Published in final edited form as:

J Acquir Immune Defic Syndr. 2013 October 1; 64(2): e13-e14. doi:10.1097/QAI.0b013e3182a0f011.

\title{
Reply to Thornton letter
}

\author{
Rebecca Plank \\ Division of Infectious Diseases, Brigham and Women's Hospital, Boston, MA
}

I write in response to inquiries raised by Dr. Thornton about our study of neonatal male circumcision in Botswana(1).

The first question raised is related to information recorded in ClinicalTrials.gov. The trial was originally designed to evaluate only the Plastibell and the Mogen clamp among 300 newborn boys (randomized 1:1 to circumcision method, 150 per arm). We learned of the availability of the newer AccuCirc device only after initiation of the original trial. After consultation with the Botswana Ministry of Health and review and approval by all relevant regulatory bodies, we added the AccuCirc arm as an amendment to our original study protocol, with a plan to circumcise an additional 150 neonates by AccuCirc (leading to a total of 450 neonates circumcised in the three arms, as reflected on ClinicalTrials.gov). Because the AccuCirc arm started enrolling only after the original two randomized Plastibell and Mogen arms had completed enrollment, the AccuCirc study results were not included in the manuscript submitted to JAIDS. The AccuCirc data have, however, been shared with the Ministry of Health in Botswana and with staff at participating local hospitals, and a manuscript summarizing the findings from the AccuCirc arm is in preparation.

The total sample size appearing in ClinicalTrials.gov includes both parents of newborn boys (because enrolled consenting parents completed questionnaires as part of this study), and boys whose parents consented to circumcision. When planning the study we anticipated that not all parents who completed the questionnaire would consent to circumcising their baby; therefore, it was necessary to estimate the number of parents who would participate in the survey (700), to achieve an enrollment of 300 neonates (therefore the initial estimate of 1000). The original two arms of the study ultimately enrolled 921 participants. The addition of the AccuCirc arm led to a revised estimate of total number of neonates and their parents (total=1250). We have updated the site to reflect the final three-arm enrollment of 1,235 participants (this includes neonates and parents).

Dr. Thornton raised questions about the deaths of three neonates who were enrolled in the study. All adverse events in the study, whether or not related to study procedures, were reported as per study protocol to the regulatory and oversight bodies who duly reviewed them. Unfortunately, neonatal and infant mortality rates remain high in low and middle

Conflicts of interest and financial disclosures: No conflicts.

Publisher's Disclaimer: This is a PDF file of an unedited manuscript that has been accepted for publication. As a service to our customers we are providing this early version of the manuscript. The manuscript will undergo copyediting, typesetting, and review of the resulting proof before it is published in its final citable form. Please note that during the production process errors may be discovered which could affect the content, and all legal disclaimers that apply to the journal pertain. 
income countries. For Botswana in 2010, UNICEF reports a neonatal mortality rate of 19/1,000 and an infant mortality rate of 36/1,000 http://www.unicef.org/infobycountry/ botswana_statistics.html). These statistics were supported by data from a recent study in Botswana of neonatal outcomes in 33,148 deliveries occurring from 2009-2011: 1.9\% of live-born infants died prior to hospital discharge (2). This sobering number does not include neonates dying after discharge from the hospital. The neonatal mortality statistics from UNICEF and from Chen et al are from sites in which neonatal male circumcision was not available. Therefore, given a neonatal mortality rate of $1.9 \%$, a total of three deaths in our study of 300 neonates is not outside of what could be expected, and no death was deemed to be circumcision-related.

One baby died with cough and gastroenteritis more than 10 weeks after being circumcised. He was born at 39 weeks estimated gestational age. The baby was circumcised on day of life 3 using a Plastibell device. There were no complications following the procedure. He was seen nearly nine weeks after the circumcision in a community outpatient clinic for cough and diarrhea. On our third attempt to contact the family after a missed 4-month follow-up visit, we learned that he had died after prolonged coughing and diarrhea, at $10^{1 / 2}$ weeks of age.

A second baby died of gastroenteritis at 28 days of age, 25 days after circumcision. The participant was born at 41 weeks estimated gestational age. The baby was circumcised on day of life 3 using a Plastibell device. There were no complications following the procedure. He was admitted to the hospital with diarrhea and vomiting on day of life 18. On admission, the study staff visited the baby and examined him. The Plastibell was already fully detached and the circumcision was completely healed and the site was clean, with no evidence of infection. The baby was treated in the hospital by hospital staff for acute gastroenteritis, dehydration and electrolyte imbalances. Unfortunately, the baby's condition never improved and he died in hospital at 28 days of age. The cause of death was documented by the treating physicians at the hospital as "Acute gastroenteritis with abdominal distention, respiratory arrest due to vomiting and query aspiration."

The last death occurred in a baby who died of suspected sepsis on day of life 3 . The baby was circumcised using a Mogen clamp on day of life 2 and discharged to home later that day. The following day he was brought to the local health center with respiratory distress, and was noted to be febrile and was transferred to the district hospital. The study team was not notified of his admission until the next morning, after he had died. The circumstances of this baby's death were reviewed in great detail with several groups, in order to obtain independent assessment of the cause of death: the hospital staff, the Botswana Ministry of Health, the Botswana Health Research and Development Committee, the Partners Institutional Review Board and our own Data Safety Monitoring Committee. All parties agreed that based upon all of the clinical data available, the most likely cause of death was neonatal sepsis or pneumonia, and that it was extremely unlikely that the baby's death was related to the circumcision procedure.

Autopsies are very rarely performed in Botswana, and were not performed in any of the three deaths in the study. Detailed diagnostic work-ups are also often not available in 
resource-limited settings, or are not performed (e.g. because a baby dies at home). Finally, prenatal screening for group B streptococcus is not routinely performed, and mothers do not receive prophylactic antibiotics.

Neonates in much of sub-Saharan Africa face the highest mortality rates in the world (3), and we agree that the exact cause of their death is often not known. We did consider but ultimately decided not to include a "no circumcision" arm in this study. We know the "background" rates of neonatal and infant mortality in Botswana, in non-circumcising populations (and these are higher than the mortality observed in our cohort). As we stated in our paper, we believe that the issue of high background rates of neonatal mortality in resource limited settings must be addressed in the context of neonatal male circumcision programs. We recommend that if a provider detects any signs or symptoms of neonatal illness, that the procedure be postponed until the neonate is deemed clinically well.

\section{Acknowledgments}

Financial support by NIH grant \# K23AI084579

\section{References}

1. Plank RM, Ndubuka NO, Wirth KE, Mwambona JT, Kebaabetswe P, Bassil B, et al. A Randomized Trial of Mogen Clamp versus Plastibell for Neonatal Male Circumcision in Botswana. J Acquir Immune Defic Syndr. 2013

2. Chen JY, Ribaudo HJ, Souda S, Parekh N, Ogwu A, Lockman S, et al. Highly active antiretroviral therapy and adverse birth outcomes among HIV-infected women in Botswana. J Infect Dis. 2012; 206(11):1695-705. [PubMed: 23066160]

3. UNICEF. Levels \& Trends in Child Mortality: Estimates Developed by the UN Inter-agency Group for Child Mortality Estimation. 2012 